\title{
Considerations of one-modulus Calabi-Yau compactifications: Picard-Fuchs equations, Kähler potentials and mirror maps
}

\author{
Albrecht Klemm ${ }^{1,2}$ \\ Physik Department T30, Technische Universität München, James-Franck-Strasse, D-8046 Garching, \\ Germany \\ Stefan Theisen ${ }^{3}$ \\ Institut für Theoretische Physik, Universität Karlsruhe, Kaiserstr. 12, D-7500 Karslruhe, Germany \\ Received 20 May 1992 \\ Accepted for publication 20 August 1992
}

\begin{abstract}
We consider Calabi-Yau compactifications with one Kähler modulus. Following the method of Candelas et al. we use the mirror hypothesis to solve the quantum theory exactly in dependence of this modulus by performing the calculation for the corresponding complex structure deformation on the mirror manifold. Here the information is accessible by techniques of classical geometry. It is encoded in the Picard-Fuchs differential equation which has to be supplemented by requirements on the global properties of its solutions.
\end{abstract}

\section{Introduction}

One of the outstanding problems in string theory is to close the gap between the formal description and classification of string vacua and their possible role in a realistic description of particle physics. Even if one finds a model with the desired particle content and gauge symmetry, one is still confronted with the problem of computing the couplings, which determine masses, mixing angles, patterns of symmetry breaking etc. These couplings will depend on the moduli of the string model, which, in the conformal field theory language correspond to the exactly marginal operators, or, in the Calabi-Yau context, to the harmonic $(1,1)$ and $(2,1)$ forms, which describe the deformation of the Kähler class and the complex structure, respectively. In $(2,2)$ compactifications, which are the ones which have been most intensively studied to date, the two types of moduli are related by world

1 Email: aklemm(ophysik.tu-muenchen.de

2 Supported by the Deutsche Forschungsgemeinschaft.

3 Email: BE01@DKAUNI2 
sheet supersymmetry to the matter fields, which transform as $\overline{27}$ and 27 of $E_{6}$. In a low energy effective field theory description, which includes all the light states, but having integrated out all heavy $\left(>m_{\text {Planck }}\right.$ ) string modes, the moduli appear as massless neutral scalar fields with perturbatively vanishing potential. This entails that the strength of the couplings, such as the Yukawa couplings, which do depend on the moduli, are undetermined. Only if the vacuum expectation value of the moduli fields is fixed by a non-perturbative potential do the couplings take fixed values, which could then be compared with experiment.

Generic string models are believed to possess duality symmetry [1], which is a discrete symmetry on moduli space that leaves the spectrum as well as the interactions invariant and whose origin is tied to the fact that strings are one-dimensional extended objects. This symmetry has been explicitly found in simple models, such as the compactification on tori and their orbifolds [2], but more recently for a specific Calabi-Yau compactification [3].

On the effective field theory level this string specific symmetry is manifest insofar as the lagrangian must be invariant [4]. This has the important consequence that the moduli dependent couplings must have definite transformation properties under transformations of the duality group *. A possible non-perturbative potential for the moduli fields must also respect this symmetry.

The problem then consists of first computing the moduli dependence of the Yukawa couplings, to find candidates for the potential for the moduli and then to look for its minimum, thus fixing the value of the Yukawa couplings.

This is a formidable task to perform for a generic string model, and has thus far only partially been done for the few simple models mentioned above. For the toroidal orbifold examples this is not too difficult if one restricts oneself to the untwisted moduli. The dependence of the couplings on the untwisted moduli can be calculated in conformal field theory [5] and the duality group of the orbifold is the subgroup of the torus duality group [1] which is compatible with the discrete group by which the orbifold is defined [2]. In simple cases the corresponding modular functions are also known. The situation for Calabi-Yau manifolds is more involved, partially because one knows the conformal field theory explicitly only at special points in moduli space, where the models coincide with the ones of Gepner [6]. Given the recent results of ref. [7] which exclude most simple toroidal orbifold models as viable candidate string vacua, one is harder pressed to develop tools to do explicit computations for more complicated compactifications. An important step in this direction has been done in ref. [3].

Above we have already mentioned the two different kinds of moduli. The moduli space is a direct product with a separate factor for the $(1,1)$ and the $(2,1)$ moduli [8]. Since in each case, due to the fact that the same $(2,2)$ superconformal

\footnotetext{
* For the simplest case where the duality group is just the modular group SL(2, $\mathbb{Z})$, they are modular forms.
} 
field theory could have been used to compactify the type II rather than the heterotic string with the former leading to $N=2$ space-time supersymmetry, the moduli space is of special Kähler type [9]. This means in particular that the Kähler metric of moduli space is completely determined in terms of two holomorphic functions, the prepotentials, one for each type of moduli. The Yukawa couplings are given by the third derivatives of the prepotentials with respect to the moduli. This entails that they do not mix the two sets of moduli and their corresponding matter fields; i.e. the Yukawa couplings of the $\overline{27}$ 's of $E_{6}$ only depend on the Kähler moduli and the couplings of the 27's depend only on the complex structure moduli. Whereas the former acquire contributions from instantons, the latter do not $[10]$ and are thus in general easier to compute.

On the conformal field theory level the 27's and $\overline{27}$ 's of $E_{6}$, and by world-sheet supersymmetry the two types of moduli, can be simply interchanged by flipping the relative sign of the left and right $\mathrm{U}(1)$ charges of the $(2,2)$ superconformal algebra [11]. On the geometrical level this corresponds to an interchange of the Hodge numbers $h_{1,1}$ and $h_{2,1}$ and thus of a change of sign of the Euler number. This so-called mirror map relates topologically distinct Calabi-Yau spaces. The mirror hypothesis states that the prepotentials for the different types of moduli are interchanged on the manifold and its mirror. Mirror symmetry thus allows one to get the instanton corrected couplings for the $(1,1)$ forms on a given Calabi-Yau manifold $\mathrm{M}$ from the couplings of the $(2,1)$ forms on its mirror $\mathrm{M}^{\prime}$, which have no instanton corrections. Following Candelas et al. we will use this strategy to compute the prepotential, Kähler potential and Yukawa couplings for the four Calabi-Yau spaces with $h_{1,1}=1$ in the lists of refs. [12,13]. Similar methods have been used in ref. [14] to compute the instanton numbers on these spaces. In one of the four cases our results differ.

The paper is organized as follows: in sect. 2 we introduce the models which we will discuss. We then (sect. 3) set up the period equations and discuss their solutions, including their monodromy properties. In sect. 4 we construct a basis for the solutions on which the monodromy transformations are integer symplectic and in terms of which the prepotential can be easily written down. We compute the Kähler metric and the invariant Yukawa couplings. In sect. 5 we perform the mirror map. In the conclusions we make some comments on the modular group and a candidate for the non-perturbative potential for the modulus. To a large extent our exposition will follow ref. [3].

\section{The models}

The simplest Calabi-Yau models are described as the vanishing locus of a quasi-homogeneous polynomial in five variables of the Fermat type $W_{0}=\sum_{i=0}^{4} x_{i}^{n_{i}}$ $=0$ which gives the embedding of the three (complex) dimensional manifold in 
weighted projective 4-space $\mathbb{p}^{4}$. Vanishing of the first Chern class and absence of singularities, whose resolution would introduce new $(1,1)$-forms, impose severe restrictions on the $n_{i}$, leaving only four manifolds which all have $h_{1,1}=1$, i.e. possess only one Kähler modulus. Characterizing these models by the integer $k$, which is defined to be the smallest common multiple of the $n_{i}$, they are (the relative factors are chosen for later convenience)

$$
\begin{array}{ll}
k=5: & \mathbf{M}=\left\{x_{i} \in \mathbb{P}(1,1,1,1,1) \mid W_{0}=x_{0}^{5}+x_{1}^{5}+x_{2}^{5}+x_{3}^{5}+x_{4}^{5}=0\right\}, \\
k=6: & \mathbf{M}=\left\{x_{i} \in \mathbb{P}(2,1,1,1,1) \mid W_{0}=2 x_{0}^{3}+x_{1}^{6}+x_{2}^{6}+x_{3}^{6}+x_{4}^{6}=0\right\} \\
k=8: & \mathbf{M}=\left\{x_{i} \in \mathbb{P}(4,1,1,1,1) \mid W_{0}=4 x_{0}^{2}+x_{1}^{8}+x_{2}^{8}+x_{3}^{8}+x_{4}^{8}=0\right\} \\
k=10: & \mathbf{M}=\left\{x_{i} \in \mathbb{P}(5,2,1,1,1) \mid W_{0}=5 x_{0}^{2}+2 x_{1}^{5}+x_{2}^{10}+x_{3}^{10}+x_{4}^{10}=0\right\} .
\end{array}
$$

These manifolds have first been found by Strominger and Witten [15]. They belong to a class of superstring compactifications whose internal space can be described at a special point of its moduli space by tensor products of minimal $(2,2)$ superconformal theories with A-type modular invariants [16], often called Gepner models. A survey of this class with A-D-E-type modular invariants can be found in * refs. $[18,13]$. The complex structure moduli of the models $(2,1)$ can be represented by those elements in the polynomial ring $\mathscr{R}=C\left[x_{i}\right] / \mathrm{d} W_{0}$ which are of the same degree as $W_{0}$. They correspond to the marginal deformations of the associated $(2,2)$ conformal field theory. One finds $h_{2,1}=101,103,149,145$ for $k=5,6,8,10$ respectively, corresponding to the Euler numbers $\chi=2\left(h_{1,1}-h_{2,1}\right)=-200,-204$, $-296,-288$.

One can now consider orbifolds of these spaces by dividing out discrete isometries which will in general not act freely. If the isometry acts like a subgroup of SU(3) the possible singularities can be resolved such that the resulting space is again of Calabi-Yau type as was conjectured in ref. [19]. This process changes the Euler number and thus also the Hodge numbers $h_{2,1}$ and $h_{1,1}$. For our investigation the most important groups are the ones which lead to the mirror configuration. They are generated by multiplying the $\mathbb{P}^{4}$ coordinates with phases: $x_{i} \rightarrow$ $x_{i} \exp \left[\left(2 \pi i / n_{i}\right) \cdot r_{i}\right]$. We abbreviate generators as $g=\left(r_{0}, \ldots, r_{4}\right)$. The condition for $g \in \mathrm{SU}(3)$ reads simply $\Sigma\left[r_{i} / n_{i}\right]=0 \bmod 1$. In appendix A we list all possible Hodge numbers which can be obtained from our models by dividing out all subgroups of the full phase symmetry group ${ }^{\star \star} \mathrm{G}$ with $\mathrm{G} \in \mathrm{SU}(3)$. The topological data can be obtained directly by a tedious calculations on the manifold, using the

* Recently more complicated models with $h_{1,1}=1$ have been constructed [17]. We will however not consider them here.

** Here we do not consider the moding of (nonabelian) permutation symmetries [20,21]. 
properties of the resolutions [22-24] but more easily by using the methods of twisting the Landau-Ginzburg models [25] or by examining the massless spectrum of the corresponding Gepner model [26,21]. The latter method gives the full partition function of the twisted theory. For the Fermat cases (A-type modular invariants) the Hodge numbers obtained this way were verified in ref. [27] by explicit construction of the geometrical resolution. In the original models we consider, all $(2,1)$-forms can be described by deformations of the defining polynomial. The orbifoldisation projects onto those $(2,1)$ forms which are invariant, their number is given in parantheses in our tables, but also introduces new ones in the twisted sectors, which cannot be represented as deformations of $\mathrm{W}_{0}$ : for a geometric representation of them see ref. [28]. In all four cases, dividing out the whole of $\mathrm{G}$, one finds that only one possible deformation survives, thus giving $h_{2,1}=1$. The resulting quotient models always appear in mirror pairs with $h_{2,1}$ and $h_{1,1}$ interchanged. See also refs. $[11,20,24]$ for the cases ${ }^{\star} k=5$; the case $k=8$ was discussed in ref. [24].

The actual order of the group action, which is very important in order to normalize our period integrals, is safely investigated on the Calabi-Yau manifold. In the $k=8$ case, e.g. the generators $g_{1}=\left(\begin{array}{llllll}0 & 1 & 0 & 0 & 7\end{array}\right), g_{2}=\left(\begin{array}{lllll}0 & 1 & 0 & 7 & 0\end{array}\right), g_{3}=$

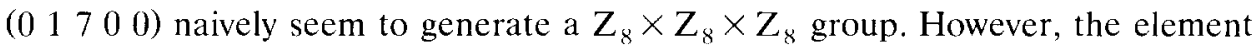
$g^{\prime}=\left(2 g_{1}\right) \times\left(2 g_{2}\right) \times\left(2 g_{3}\right) \simeq(0,-2,-2,-2,-2)$ generates a $Z_{4}$ subgroup which operates trivially on the coordinates, because of the equivalence relation of the $\mathbb{P}(4,1,1,1,1)$ we have $\left(x_{0},-i x_{1},-i x_{2},-i x_{3},-i x_{4}\right) \simeq\left(x_{0}, x_{1}, x_{2}, x_{3}, x_{4}\right)$. Hence the actual group acts as a $Z_{8} \times Z_{8} \times Z_{2}$. In the Gepner or Landau-Ginzburg model language this corresponds to the fact that the aforementioned $Z_{4}$ acts a subgroup of the group by which it is divided out in order to implement the GSO projection. The actual groups which generate the mirror configurations are thus $\mathrm{G}=\mathrm{Z}_{5}^{3}, \mathrm{Z}_{3} \times \mathrm{Z}_{6}^{2}, \mathrm{Z}_{8}^{2} \times \mathrm{Z}_{2}$ and $\mathrm{Z}_{10}^{2}$ with Ord $\mathrm{G}=5^{3}, 3 \cdot 6^{2}, 2 \cdot 8^{2}$ and $10^{2}$ in the four cases, respectively. We observe that (Ord G) $\prod_{i=0}^{4} \nu_{i}=k^{3}$, where the $\nu_{i}=k / n_{i}$ are the weights of $\mathbb{P}^{4}$ coordinates which satisfy $\sum_{i=0}^{4} \nu_{i}=k$. We then get for the deformed polynomials

$$
W=W_{0}-k \alpha x_{0} x_{1} x_{2} x_{3} x_{4}
$$

where the perturbation can always be cast into the indicated form by the use of the equations of motion $\partial_{i} W=0$. The constants in (2.1) and (2.2) have been chosen such that $\alpha^{k}=1$ are nodes in all four cases. The elements of the rings $\mathscr{R}$ are now also restricted by the discrete symmetries. In the case where one divides out all of $\mathrm{G}, \mathscr{R}$ consists of only the elements $\left(x_{0} x_{1} x_{2} x_{3} x_{4}\right)^{\lambda}$ with $\lambda=0,1,2,3$. Besides having nodes at $\alpha^{k}=1$ the manifolds become singular at $\alpha \rightarrow \infty$.

\footnotetext{
* For the $k=10$ case the list given in ref. [11] is incomplete.
} 


\section{The Picard-Fuchs equations and their solutions}

To summarize the results of refs. [29-31] the Picard-Fuchs or period equations are differential equations satisfied by the expression $w=\int q(\alpha) / W(\alpha)$ where the integral is in the embedding space and allows integration by parts with respect to the coordinates of $\mathbb{P}^{4}$. The holomorphic function $q(\alpha)$ reflects the gauge freedom in the definition of the holomorphic three-form. For the purpose of deriving the period equation, it is most convenient to set $q(\alpha)=1$. Differentiating $\lambda$ times with respect to $\alpha$ produces terms of the form $\int\left[\left(x_{1} x_{2} x_{3} x_{4} x_{5}\right)^{\lambda} / W^{\lambda+1}(\alpha)\right]$. The $\lambda=4$ term, which is the first to produce an integrand whose numerator is no longer in the ring $\mathscr{R}$, can be expressed, using the equations $\partial W / \partial x_{i}=\ldots$ and integration by parts, in terms of lower derivatives. The computation is straightforward and produces *

$$
\begin{array}{ll}
k=5: \quad & \left(1-\alpha^{5}\right) w^{(\mathrm{iv})}-10 \alpha^{4} w^{\prime \prime \prime}-25 \alpha^{3} w^{\prime \prime}-15 \alpha^{2} w^{\prime}-\alpha w=0 \\
k=6: \quad & \alpha^{2}\left(1-\alpha^{6}\right) w^{(\mathrm{iv})}-2 \alpha\left(1+5 \alpha^{6}\right) w^{\prime \prime \prime}+\left(2-25 \alpha^{6}\right) w^{\prime \prime} \\
& -15 \alpha^{5} w^{\prime}-\alpha^{4} w=0 \\
k=8: \quad & \alpha^{3}\left(1-\alpha^{8}\right) w^{(\mathrm{iv})}-\alpha^{2}\left(6+10 \alpha^{8}\right) w^{\prime \prime \prime}+5 \alpha\left(3-5 \alpha^{8}\right) w^{\prime \prime} \\
& -15\left(1+\alpha^{8}\right) w^{\prime}-\alpha^{7} w=0 \\
k=10: \quad & \alpha^{3}\left(1-\alpha^{10}\right) w^{(\mathrm{iv})}-10 \alpha^{2}\left(1+\alpha^{10}\right) w^{\prime \prime \prime}+5 \alpha\left(7-5 \alpha^{10}\right) w^{\prime \prime} \\
& -5\left(7+3 \alpha^{10}\right) w^{\prime}-\alpha^{9} w=0
\end{array}
$$

A fundamental system of solutions may be obtained following the method of Froebenius for ordinary differential equations with regular singular points [33] which are here $\alpha=0, \alpha=\infty$ and $\alpha^{k}=1$. The solutions of the indicial equations at the three singular points are $\rho=(0,1,2,3)_{k=5},(0,1,3,4)_{k=6},(0,2,4,6)_{k=8}$, $(0,2,6,8)_{k=10}$ for $\alpha=0, \rho=\left(0,1_{2}, 2\right)$ for $\alpha^{k}=1$ and $\rho=0_{4}$ for $\alpha=\infty$. The subscripts denote the multiplicities of the solutions. It follows from the general theory that at $\alpha=\infty$ there is one solution given as a pure power series and three containing logarithms (with powers 1,2 and 3, respectively). At $\alpha=0$, all four solutions are pure power series as one sees, e.g. by noting that we can rewrite the differential equation in terms of the variables $\alpha^{k}$, for which the solutions of the indicial equation would no longer differ by integers. The point $\alpha=1$ needs some care ${ }^{\star}$. There is one power series solution with index $\rho=2$ and at least one

\footnotetext{
* Manifolds having the mirror Hodge diamond w.r.t. the models in (2.1) can be found in the lists of refs. [12,21]. As has been checked in a few examples [32] the Picard-Fuchs equations for the only complex structure deformation here turn out to be the same as in (3.1).

** The other solutions of $\alpha^{k}=1$ are treated similarly.
} 
logarithmic solution for $\rho=1$. Making a power series ansatz for $\rho=0$ one finds that the first three coefficients are arbitrary which means that there is one power series solution for each $\rho$. One also easily checks that in the second solution to $\rho=1$ the logarithm is multiplied by a linear combination of the power series solutions with indices 1 and 2 . To summarize, the periods of the manifolds have logarithmic singularities at the values of $\alpha$ corresponding to the node $\left(\alpha^{k}=1\right)$ and to the singular manifold $(\alpha=\infty)$. We will thus get non-trivial monodromy about these points.

The power series solution around $\alpha=\infty$ is easy to find by making a general ansatz and solving the recursion relation for the coefficients. One finds

$$
w_{0}(\alpha)=\frac{1}{\alpha} \sum_{m=0}^{\infty} \frac{(k m) !}{\prod_{i=0}^{4}\left(\nu_{i} m\right) !}(\gamma \alpha)^{-k m}
$$

where $\gamma=k \prod_{i=0}^{4}\left(\nu_{i}\right)^{-\nu_{i} / k}$. The other solutions around $\alpha=\infty$ contain logarithms. We will find them below. For later convenience we will redefine the periods to get rid of the factor $\alpha^{-1}$. This corresponds to the gauge transformation $q(\alpha)=1 \rightarrow$ $q(\alpha) \propto \alpha$. This only affects the exponents at $\alpha=0$ which are shifted by +1 . To get the solutions around $\alpha=0$ we first analytically continue above solution. If $0 \leqslant$ $\arg \alpha<2 \pi / k$, we have

$$
w_{0}(\alpha)=\frac{1}{2 \pi i} \int_{C} \mathrm{~d} s \frac{\Gamma(1+k s)}{\prod_{i=0}^{4} I\left(1+\nu_{i} s\right)} \frac{\pi \mathrm{e}^{i \pi s}}{\sin (\pi s)}(\gamma \alpha)^{-k s} .
$$

For $|\alpha|>1$ we get a convergent expression if we choose the contour to enclose the points $\alpha=n=0,1,2 \ldots$ which are zeros of $\sin (\pi s)$, in a clockwise direction and we recover (3.2). For $|\alpha|<1$ we close the contour so as to enclose the points $-n / k, n=1,2 \ldots$ which are the poles of $\Gamma(k s+1)$ with residues $(-1)^{n+1} /(k \Gamma(n))$. One finds

$$
w_{0}(\alpha)=-\frac{\pi}{k} \sum_{n=1}^{\infty} \frac{1}{\Gamma(n) \prod_{i=0}^{4} \Gamma\left(1-(n / k) \nu_{i}\right)}-\frac{\mathrm{e}^{i(\pi / k)(k-1) n}}{\sin (\pi n / k)}(\gamma \alpha)^{n} .
$$

All solutions at $\alpha=0$ are given by power series and one readily sees that the functions $w_{j}(\alpha):=w_{0}\left(\beta^{i} \alpha\right),(j=0,1, \ldots, k-1)$ with $\beta=\mathrm{e}^{2 \pi i / k}$ are also solutions. These are however not all linearly independent. There are $k-4$ linear relations. One possible choice is $\sum_{j=0}^{4} w_{j}=0$ for $k=5, w_{0}+w_{2}+w_{4}=w_{1}+w_{3}+w_{5}=0$ for $k=6, w_{i}+w_{i+4}=0,(i=0,1,2,3)$ for $k=8, w_{i}+w_{i+5}=0,(i=0,1,2,3,4)$ and $w_{0}+w_{2}+w_{3}+w_{4}+w_{5}=0$ for $k=10$. Below we will use the functions $w_{0}, w_{1}, w_{2}$ and $w_{k-1}$ as a basis. 
To get all the solutions for $\alpha=\infty$ we first express the $w_{j}$ in terms of four linearly independent solutions which we then analytically continue from $|\alpha|<1$ to $|\alpha|>$ 1. Changing the summation index to $n=k N+l, N=0,1,2, \ldots, l=1, \ldots, k-1$, one obtains

$$
w_{j}(\alpha)=-\frac{1}{16 k \pi^{4}} \sum_{l} \beta^{j l} \frac{\prod_{i=0}^{4}\left(\beta^{l \nu_{i}}-1\right)}{\beta^{l}-1} \tilde{w}_{l}(\alpha),
$$

with

$$
\begin{aligned}
\tilde{w}_{l}(\alpha) & =\sum_{N=0}^{\infty} \frac{\prod_{i=0}^{4} \Gamma\left(\nu_{i}(N+l / k)\right)}{\Gamma(k N+l)}(\gamma \alpha)^{k N+l} \\
& =-\int_{\mathrm{C}} \frac{\mathrm{d} s}{\mathrm{e}^{2 \pi i s}-1} \frac{\prod_{i=0}^{4} \Gamma\left(\nu_{i}(s+l / k)\right)}{\Gamma(k s+l)}(\gamma \alpha)^{k s+l},
\end{aligned}
$$

where to recover (3.5) one has to choose the contour so as to enclose the poles of $\left(\mathrm{e}^{2 \pi i s}-1\right)^{-1}$. Note that in eq. (3.5) there are only four non-zero terms in each sum, namely for $l=(1,2,3,4)_{k=5}, l=(1,2,4,5)_{k=6}, l=(1,3,5,7)_{k=8}$ and $l=$ $(1,3,7,9)_{k=10}$. To get the solutions for $|\alpha|>1$ one chooses the contour to surround the poles of the second factor of the integrand which has quadruple poles at $s=-N-l / k$ for $N=0,1, \ldots$ Evaluating the residues at the poles is straightforward. Expanding $\alpha^{k s+l}$ around the poles produces up to three powers of $\log \alpha$. Collecting terms one finds

$$
w_{j}(\alpha)=-\frac{1}{(2 \pi i)^{3}} \frac{1}{\prod_{i=0}^{4} \nu_{i}} \sum_{r=0}^{3} \log ^{r}(\gamma \alpha) \sum_{N=0}^{\infty} \frac{(k N) !}{\prod_{i=0}^{4}\left(\nu_{i} N\right) !} b_{j r N}(\gamma \alpha)^{-k N}
$$

where

$$
\begin{aligned}
b_{j 0 N}= & (2 \pi i)^{3}\left(S_{j 4}-\prod_{i=0}^{4} \nu_{i}\right)+(2 \pi i)^{2}(2 \pi i+k \phi(N)) S_{j 3} \\
& +\frac{2 \pi i}{24}\left((2 \pi i)^{2}\left(4+k^{2}-\sum_{i=0}^{4} \nu_{i}^{2}\right)+12(2 \pi i) k \phi(N)\right. \\
& \left.+12\left(k^{2} \phi^{2}(N)-k \phi^{\prime}(N)\right)\right) S_{j 2}
\end{aligned}
$$




$$
\begin{aligned}
+ & \frac{k}{24}\left((2 \pi i)^{2}\left(k^{2}-\sum_{i=0}^{4} \nu_{i}^{2}\right) \phi(N)+4 k^{2} \phi^{3}(N)\right. \\
& \left.-12 k \phi(N) \phi^{\prime}(N)+4 \phi^{\prime \prime}(N)\right) S_{j 1}, \\
b_{j 1 N}= & k(2 \pi i)^{2} S_{j 3}+\frac{k}{2}(2 \pi i)((2 \pi i)+2 k \phi(N)) S_{j 2} \\
& +\frac{k}{24}\left((2 \pi i)^{2}\left(k^{2}-\sum_{i=0}^{4} \nu_{i}^{2}\right)+12 k^{2} \phi^{2}(N)-12 k \phi^{\prime}(N)\right) S_{j 1}, \\
b_{j 2 N}= & \frac{k^{2}}{2}\left((2 \pi i) S_{j 2}+k \phi(N) S_{j 1}\right), \\
b_{j 3 N}= & \frac{k^{3}}{6} S_{j 1} .
\end{aligned}
$$

Here we have defined

$$
\begin{aligned}
\phi_{k}(N) & =\frac{1}{k} \sum_{i=0}^{4} \nu_{i} \psi\left(1+\nu_{i} N\right)-\psi(1+k N), \quad \psi(x)=\mathrm{d} \log \Gamma(x) / \mathrm{d} x, \\
S_{j m} & =\sum_{l=0}^{k-1} \beta^{l(j+1)} \frac{\prod_{i=0}^{4}\left(\beta^{\nu_{i}}-1\right)}{\left(\beta^{l}-1\right)^{m+1}} .
\end{aligned}
$$

We now discuss the monodromy of the solutions. Under the transformation $\alpha \rightarrow \beta \alpha$, the solutions behave as $w_{j}(\beta \alpha) \rightarrow w_{j+1}(\alpha)$ and the corresponding monodromy matrix $a$ is cyclic of order $k$. From our discussion above of the solutions of the period equations around $\alpha=1$ we conclude that the $w_{j}$, when continued to the region $|\alpha-1|<1$, must be of the form

$$
w_{j}(\alpha)=\frac{1}{2 \pi i} c_{j} \tilde{w}(\alpha) \log (\alpha-1)+\text { regular }
$$

where $\tilde{w}(\alpha)$ is a particular combination of the power series solutions with indices 1 and 2. In fact, we can express it in terms of the $w_{j}$ as follows [3]:

$$
\tilde{w}(\alpha)=-\frac{1}{c_{1}}\left(w_{1}(\alpha)-w_{0}(\alpha)\right) .
$$


Eq. (3.8) then says that under $(\alpha-1) \rightarrow \mathrm{e}^{2 \pi i}(\alpha-1)$ the solutions transform as

$$
w_{j}(\alpha) \rightarrow w_{j}(\alpha)-\frac{c_{j}}{c_{1}}\left(w_{1}(\alpha)-w_{0}(\alpha)\right)
$$

We thus find that the monodromy matrix $t$ around $\alpha=1$ is not cyclic of any finite order. To determine the coefficients $c_{j}$ we normalize $\tilde{w}(\alpha)=\mu(\alpha-1)+\mathrm{O}((\alpha-$ $\left.1)^{2}\right)$ such that $\mu c_{1}=1 . \mu c_{j}$ is then the coefficient of the logarithm of $\mathrm{d} w_{j} / \mathrm{d} \alpha$ as $\alpha \rightarrow 1$. Using the explicit expressions for $w_{j}$ [eq. (3.5)] we find $\mu=$ $-\left(1 / 2 \pi_{i}\right) k^{3 / 2}\left(\prod_{i=0}^{4} \nu_{i}\right)^{-1 / 2}$ and

$$
c_{j}=\frac{1}{k} S_{j-1,0} .
$$

The $c_{j}$ are all integers. In all cases $c_{0}=c_{1}$, which is necessary for (3.9) to be free from logarithms. Monodromy transformations about the points $\beta^{l}$ follow simply from a composition of the transformations $a$ and $t$. The monodromy * about $\alpha=\beta^{l}$ is then $a^{-l} t a^{l}$. Finally, the monodromy matrix $s$ around $\alpha=\infty$ follows from the fact that the product of the monodromy matrices around all singular points must be the identity and that the monodromy around $\alpha=0$ is trivial. Thus $s=\left((a t)^{-1}\right)^{k}$.

\section{The periods in a symplectic basis and their monodromy}

In order to get the prepotential $\mathscr{G}$ from the solutions of the period equations, we look for a basis in which the monodromy acts as $\operatorname{Sp}(4 ; \mathbb{Z})$ transformations. $\mathscr{G}$ is then given as $\mathscr{G}=\frac{1}{2} \mathscr{G}_{a} z^{a}$, where the periods $\mathscr{G}_{a}$ and $z^{a}\left(a=0, \ldots, h_{2,1}\right)$ are the integrals of the holomorphic three form over a symplectic basis for the homology group $\mathrm{H}_{3}(\mathbb{Z}) . \mathscr{G}$ is homogeneous of degree two in the homogeneous coordinates $z^{a}$ and $\mathscr{G}_{a}=\partial \mathscr{G} / \partial z^{a}$. In appendix B we directly identify two of the solutions of the Picard-Fuchs equation with $\mathscr{G}_{2}$ and $z^{2}$. With the gauge choice $q(\alpha)=k \alpha$ we find $\mathscr{G}_{2}(\alpha)=\lambda_{1} w_{0}(\alpha)$ with $\lambda_{1}=(2 \pi i)^{3} /$ Ord G. $z^{2}(\alpha)$ is a solution which, around $\alpha=1$, is a pure power series with index 1, proportional to $\tilde{w}(\alpha)$ in eq. (3.10) and is given, to leading order as $z^{2}(\alpha)=\lambda_{2}(\alpha-1)+\mathrm{O}\left((\alpha-1)^{2}\right)$ with $\lambda_{2}=\left(4 \pi^{2} / k^{3 / 2}\right)\left(\prod \nu_{i}\right)^{1 / 2}$. Then $\lambda_{2} / \lambda_{1}=\mu$ and the monodromy coefficients $c_{j}$ become integers.

We now define two period vectors [3],

$$
\Pi^{\prime}=\left(\mathscr{G}_{1}, \mathscr{G}_{2}, z^{1}, z^{2}\right)^{\mathrm{T}}
$$

and

$$
w=-\frac{(2 \pi i)^{3}}{\operatorname{Ord} \mathrm{G}}\left(w_{2}, w_{1}, w_{0}, w_{k-1}\right)^{\mathrm{T}}
$$

\footnotetext{
* Note that if one represents, as we do, the monodromy group on the fundamental system by matrix multiplication from the left, the matrices form an anti-homomorphism of the monodromy group.
} 
Since they both represent a fundamental set of solutions of the period equations, they must be related by a linear transformation which we call $m$. From the above identification of two of the components of $\Pi$ with elements of $w$, we already know the second and last row of the matrix $m$. Since the cycles dual to the periods $\mathscr{G}_{1}$ and $z^{1}$ are remote from the node at $\alpha=1$, they must be free from logarithms. This gives a constraint on the first and third row of $m$. We then fix the remaining six components of $m$ by requiring that under $\alpha \rightarrow \beta \alpha$, which was represented on the basis $w_{i}$ by the matrix $a$, the vector $\Pi^{\prime}$ transforms under an integer symplectic transformation $A$; i.e. we determine $m$ such that $A=\operatorname{mam}^{-1} \in \mathrm{SP}(4 ; \mathbb{Z})$. ${ }^{*}$ This does not yet fix the matrix $\mathrm{m}$ uniquely. Having defined the periods $\mathscr{G}_{2}$ and $z^{2}$, the remaining two can only be fixed by the above requirement up to a $\operatorname{SP}(2 ; \mathbb{Z}) \subset$ $\mathrm{SP}(4 ; \mathbb{Z})$ transformation. With a suitable choice we obtain the results given below. We have also recorded the transformation matrices $S=\mathrm{msm}^{-1}$ for the monodromy about $\alpha=\infty$ in the symplectic basis. (For completeness we also record the result for $k=5$ already given in ref. [3].)

$$
\begin{aligned}
& k=5: \quad m=\left(\begin{array}{rrrr}
-\frac{3}{5} & -\frac{1}{5} & \frac{21}{5} & \frac{8}{5} \\
0 & 0 & -1 & 0 \\
-1 & 0 & 8 & 3 \\
0 & 1 & -1 & 0
\end{array}\right), \quad A=\left(\begin{array}{rrrr}
-9 & -3 & 5 & 3 \\
0 & 1 & 0 & -1 \\
-20 & -5 & 11 & 5 \\
-15 & 5 & 8 & -4
\end{array}\right) \text {, } \\
& S=\left(\begin{array}{rrrr}
51 & 90 & -25 & 0 \\
0 & 1 & 0 & 0 \\
100 & 175 & -49 & 1 \\
-75 & -125 & 35 & 1
\end{array}\right) \\
& k=6: \quad m=\left(\begin{array}{rrrr}
-\frac{1}{3} & -\frac{1}{3} & \frac{1}{3} & \frac{1}{3} \\
0 & 0 & -1 & 0 \\
-1 & 0 & 3 & 2 \\
0 & 1 & -1 & 0
\end{array}\right), \quad A=\left(\begin{array}{rrrr}
1 & -1 & 0 & 1 \\
0 & 1 & 0 & -1 \\
-3 & -3 & 1 & 3 \\
-6 & 4 & 1 & -3
\end{array}\right) \text {, } \\
& S=\left(\begin{array}{rrrr}
1 & 6 & 0 & 0 \\
0 & 1 & 0 & 0 \\
18 & 81 & 1 & 0 \\
-27 & -129 & -6 & 1
\end{array}\right) \\
& k=8: \quad m=\left(\begin{array}{rrrr}
-\frac{1}{2} & -\frac{1}{2} & \frac{1}{2} & \frac{1}{2} \\
0 & 0 & -1 & 0 \\
-1 & 0 & 3 & 2 \\
0 & 1 & -1 & 0
\end{array}\right), \quad A=\left(\begin{array}{rrrr}
1 & -1 & 0 & 1 \\
0 & 1 & 0 & -1 \\
-2 & -2 & 1 & 2 \\
-4 & 4 & 1 & -3
\end{array}\right) \text {, }
\end{aligned}
$$

\footnotetext{
* A matrix $M$ is symplectic if it satisfies $M \Sigma^{\prime} M^{\mathrm{T}}=\mathbf{\Sigma}$, where $\mathbf{\Sigma}=\left({ }_{-10}\right)$ is the symplectic metric.
} 


$$
\begin{aligned}
S & =\left(\begin{array}{rrrr}
1 & 8 & 0 & 0 \\
0 & 1 & 0 & 0 \\
16 & 88 & 1 & 0 \\
-40 & -200 & -8 & 1
\end{array}\right), \\
k=10: \quad m & =\left(\begin{array}{rrrr}
0 & 1 & 1 & 1 \\
0 & 0 & -1 & 0 \\
1 & 0 & 0 & -1 \\
0 & 1 & -1 & 0
\end{array}\right), \quad A=\left(\begin{array}{rrrr}
1 & 0 & 1 & 0 \\
0 & 1 & 0 & -1 \\
0 & 1 & 1 & -1 \\
1 & 3 & 1 & -2
\end{array}\right), \\
& S=\left(\begin{array}{rrrr}
1 & 55 & -10 & 0 \\
0 & 1 & 0 & 0 \\
0 & -10 & 1 & 0 \\
-10 & -195 & 45 & 1
\end{array}\right) .
\end{aligned}
$$

The matrices $A$ satisfy $A^{4}=-1$ and $A^{5}=-1$ in the cases $k=8$ and $k=10$, respectively.

The matrix $T=m m^{-1}$, which describes the monodromy around $\alpha=1$, is the same in all cases:

$$
T=\left(\begin{array}{llll}
1 & 0 & 0 & 0 \\
0 & 1 & 0 & 1 \\
0 & 0 & 1 & 0 \\
0 & 0 & 0 & 1
\end{array}\right)
$$

We have verified that the components of $\Pi^{\prime}$ pass the consistency check of ref. [3], $W_{1}=0$ where $W_{k}=z^{a} \partial_{\alpha}^{k} \mathscr{G}_{a}-\mathscr{G}_{a} \partial_{a}^{k} z^{a}$.

Let us now turn to the quantities which are relevant for the low energy effective lagrangian of the string theory compactified for the Calabi-Yau spaces under considerations. The Kähler potential on the one-dimensional moduli space for the complex structure modulus is given in terms of the prepotential:

$$
\mathrm{e}^{-K}=-i\left(z^{a} \dot{\partial}_{\overline{\bar{z}}} \overline{\mathscr{G}}-\bar{z}^{a} \partial_{z^{a}} \mathscr{G}\right)=-i \Pi^{\dagger \dagger} \Sigma \Pi^{\prime} .
$$

We give the results in the limits $\alpha \rightarrow \infty$ for which we use the solutions in the form (3.7) and for $\alpha \rightarrow 0$ using (3.4): $\alpha \rightarrow \infty$ :

$$
\begin{aligned}
& \mathrm{e}^{-K} \simeq \frac{(2 \pi)^{3}}{\text { Ord G }}\left(\frac{4 k}{3} \log ^{3}|\gamma \alpha|+\frac{2}{3 k^{2}}\left(k^{3}-\sum_{i=0}^{4} \nu_{i}^{3}\right) \zeta(3)\right) \\
& g_{\alpha \bar{\alpha}} \simeq \frac{3}{4|\alpha|^{2} \log ^{2}|\gamma \alpha|}\left(1+\frac{2\left(\sum_{i=0}^{4}\left(\nu_{i} / k\right)^{3}-1\right) \zeta(3)}{-\log ^{3}|\gamma \alpha|}\right)
\end{aligned}
$$


In terms of the variable $t \propto i \log (\gamma \alpha)$ the leading behaviour is $g_{t \bar{t}} \simeq-3 /(t-\bar{t})^{2}$ which is the metric for the upper half-plane with curvature $R=-4 / 3$ :

$\alpha \rightarrow 0^{\star}$ :

$$
\begin{aligned}
& \mathrm{e}_{k=5}^{-K}=\frac{(2 \pi)^{3}}{5^{5}} \frac{\Gamma^{5}\left(\frac{1}{5}\right)}{\Gamma^{5}\left(\frac{4}{5}\right)}|\alpha|^{2}+\mathrm{O}\left(|\alpha|^{4}\right) ; \\
& \mathrm{e}_{k=6}^{-K}=\frac{2^{13 / 3} \pi^{8}}{3^{11 / 2} \Gamma^{2}\left(\frac{2}{3}\right) \Gamma^{8}\left(\frac{5}{6}\right)}|\alpha|^{2}+\mathrm{O}\left(|\alpha|^{4}\right), \\
& \mathrm{e}_{k=8}^{-K}=\frac{\pi^{7}}{128} \frac{\cot ^{2}\left(\frac{\pi}{8}\right)}{\Gamma^{8}\left(\frac{7}{8}\right)}|\alpha|^{2}+\mathrm{O}\left(|\alpha|^{6}\right), \quad \mathrm{e}_{k=10}^{-K} \simeq 104.61|\alpha|^{2}+\mathrm{O}\left(|\alpha|^{6}\right) ; \quad(4.4) \\
& g_{\alpha \bar{\alpha}}^{k=5}=25\left(\frac{\Gamma\left(\frac{4}{5}\right) \Gamma\left(\frac{2}{5}\right)}{\Gamma^{3}\left(\frac{1}{5}\right) \Gamma\left(\frac{3}{5}\right)}\right)^{5}+\mathrm{O}\left(|\alpha|^{2}\right), \quad g_{\alpha \bar{\alpha}}^{k=6}=\frac{3 \Gamma^{8}\left(\frac{5}{6}\right)}{2^{2 / 3} \pi^{2} \Gamma^{4}\left(\frac{2}{3}\right)}+\mathrm{O}\left(|\alpha|^{2}\right), \\
& g_{\alpha \bar{\alpha}}^{k=8}=\frac{64\left(3-2^{3 / 2}\right)^{2} \Gamma^{8}\left(\frac{7}{8}\right)}{\Gamma^{8}\left(\frac{5}{8}\right)}|\alpha|^{2}+\mathrm{O}\left(|\alpha|^{8}\right), \quad g_{\alpha \bar{\alpha}}^{k=10} \simeq 0.170|\alpha|^{2}+\mathrm{O}\left(|\alpha|^{6}\right) .
\end{aligned}
$$

The Yukawa couplings are $\kappa_{\alpha \alpha \alpha}=\int_{\mathrm{M}^{\prime}} \Omega \wedge \partial^{3} \Omega / \partial \alpha^{3}$. Decomposing the holomorphic three form $\Omega$ as in appendix B and using

$$
\int_{\mathrm{M}^{\prime}} \alpha_{\mathrm{a}} \wedge \beta^{\mathrm{b}}=\delta_{a}^{b}, \quad \int_{\mathrm{M}^{\prime}} \alpha_{a} \wedge \alpha_{b}=\int_{\mathrm{M}^{\prime}} \beta^{a} \wedge \beta^{b}=0
$$

one finds $\kappa_{\alpha \alpha \alpha}=\mathrm{W}_{3}$. This form for $\kappa_{\alpha \alpha \alpha}$ is not the most convenient one and we will use it only to fix the overall normalization by evaluating $W_{3}$ in the limit $\alpha \rightarrow 0$. Another relation satisfied by $\kappa_{\alpha \alpha \alpha}$ has been found in ref. [3]. There it is shown that the Yukawa coupling satisfies the first order differential equation $\partial_{\alpha} \kappa_{\alpha \alpha \alpha}$ $+\frac{1}{2} C_{3} \kappa_{\alpha \alpha \alpha}=0$ where $C_{3}$ is the coefficient of the third derivative in the PicardFuchs equation with the coefficient of the highest derivative normalized to one. Using the form of the Picard-Fuchs equation as given in eq. (3.1) we easily derive $\kappa_{\alpha \alpha \alpha} \alpha \alpha^{k-5} /\left(1-\alpha^{k}\right)$. Taking now into account that below eq. (3.2) we have made a gauge transformation by rescaling the holomorphic three form by a factor

* For $k=10$ the analytic expressions are rather cumbersome so we decided to give only the numerical values. 
$q(\alpha) \alpha \alpha$, which affects the Yukawa coupling as $\kappa_{\alpha \alpha \alpha} \rightarrow q^{2}(\alpha) \kappa_{\alpha \alpha \alpha}$ and fixing the overall normalization as described above, we finally obtain

$$
\kappa_{\alpha \alpha \alpha \alpha}=\frac{(2 \pi i)^{3}}{\operatorname{Ord} \mathrm{G}} \frac{k \alpha^{k-3}}{1-\alpha^{k}} .
$$

The invariant Yukawa couplings are defined as

$$
\mathscr{Y}_{\text {inv }}=g_{\alpha \bar{\alpha}}^{-3 / 2} \mathrm{e}^{K}\left|\kappa_{\alpha \alpha \alpha \alpha}\right| .
$$

They correspond to a canonically normalized kinetic energy of the matter fields (hence the factor $g_{\alpha \bar{\alpha}}^{-3 / 2}$ ) and are invariant under Kähler gauge transformations (hence the factor $\mathrm{e}^{K}$ ). In the limits considered above we find for the leading terms of the Yukawa couplings of the one multiplet of 27 of $E_{6}$ :

$\alpha \rightarrow \infty$ :

$$
\mathscr{Y}_{\text {inv }}=\frac{2}{\sqrt{3}} \forall k
$$

$\alpha \rightarrow 0$ :

$$
\begin{aligned}
& \mathscr{Y}_{\text {inv }}^{k=5}=\left(\frac{\Gamma^{3}\left(\frac{3}{5}\right) \Gamma\left(\frac{1}{5}\right)}{\Gamma^{3}\left(\frac{2}{5}\right) \Gamma\left(\frac{4}{5}\right)}\right)^{5 / 2}+\mathrm{O}\left(|\alpha|^{2}\right), \quad \mathscr{Y}_{\text {inv }}^{k=6}=2^{4 / 3}|\alpha|+\mathrm{O}\left(|\alpha|^{3}\right), \\
& \mathscr{Y}_{\text {inv }}^{k=8}=\frac{\Gamma^{6}\left(\frac{5}{8}\right) \Gamma^{2}\left(\frac{1}{8}\right)}{\Gamma^{6}\left(\frac{3}{8}\right) \Gamma^{2}\left(\frac{7}{8}\right)}+\mathrm{O}\left(|\alpha|^{2}\right), \quad \mathscr{Y}_{\text {inv }}^{k=10}=3.394|\alpha|^{2}+\mathrm{O}\left(|\alpha|^{6}\right) .
\end{aligned}
$$

For $k=5,8$ the nonvanishing couplings coincide with the values of the corresponding Gepner models, which can be calculated using the relation [34] between the operator product coefficients of the minimal $(n=2)$ superconformal models and the known ones of the su(2) Wess-Zumino-Witten theories. In the $k=6,10$ cases the additional U(1) selection rules at the Gepner point exclude the coupling, which is allowed for generic values of the modulus.

\section{The mirror maps}

So far we have only considered the complex structure modulus and the Kähler metric and Yukawa coupling on the mirror manifold $\mathbf{M}^{\prime}$ of the original manifold M. Here all quantities could be obtained from the solutions of the Picard-Fuchs equations. The couplings involving the $(1,1)$ sector of moduli space of the original manifold cannot be obtained from the periods on that manifold, but rather from the periods of the mirror manifold via the mirror map briefly described in sect. 1 . 
The $(1,1)$ sector of the original manifold is also described by a holomorphic function, denoted by $\mathscr{F}$. It is also of the form $\mathscr{F}=\frac{1}{2} \omega^{a} \mathscr{F}_{a}$, which is homogeneous of degree two in the $\omega^{a}$ and thus $\mathscr{F}_{a}=\partial \mathscr{F} / \partial \omega^{a}$.

The large radius limit of $\mathscr{F}$ is known; it takes the simple form

$$
\widetilde{F}_{0}=-\frac{\kappa_{0}}{6} \frac{\left(\omega^{1}\right)^{3}}{\omega^{2}}=-\frac{\kappa_{0}}{6}\left(\omega^{2}\right)^{2} t^{3}=\left(\omega^{2}\right)^{2} \tilde{\mathscr{F}}_{0},
$$

where we have introduced the inhomogeneous coordinate $t=\omega^{1} / \omega^{2} \cdot \kappa_{0}=-\partial_{f}^{3} \tilde{F}_{0}$ is the infinite radius limit of the Yukawa coupling and is given by an intersection number. In general, if we define $\mathscr{F}=\left(\omega^{2}\right)^{2} \tilde{F}$ the Yukawa coupling $\kappa$ and the Kähler potential are given in terms of $\tilde{\mathscr{F}}$ as follows:

$$
\begin{aligned}
\kappa_{t t t} & =-\left.\partial_{\omega}^{3} \mathscr{F}\right|_{\omega}{ }^{2}=1 \\
K & =-\log \left((t-\bar{t})\left(\partial_{t} \tilde{\mathscr{F}}-\bar{\partial}_{i} \tilde{\tilde{F}}\right)-2(\tilde{\mathscr{F}}-\overline{\tilde{F}})\right) .
\end{aligned}
$$

(Here $K$ differs from the one given in terms $\mathscr{F}$ (cf. below) by a Kähler transformation.)

Let us briefly recall how to compute the intersection numbers [35]. The Kähler form $\tilde{J}$ of a complete intersection Calabi-Yau M, which is defined by a polynom constraint of degree $\operatorname{deg}(p)$ in a weighted projective space $\mathbb{P}$ is given as the pullback of the Kähler form $J$ of the latter one, by $\tilde{J}=i^{*} J$, where $i$ : $\mathrm{M} \hookrightarrow \mathbb{P}$ is the inclusion map. The topological three point function $\kappa_{0}(\tilde{J}, \tilde{J}, \tilde{J})$ is then most easily computed by lifting the integration over $M$ to the ambient space $\mathbb{P}\left(\nu_{0}, \ldots, \nu_{n}\right)$

$$
\kappa_{0}(\tilde{J}, \tilde{J}, \tilde{J})=\int_{\mathrm{M}} \tilde{J} \wedge \tilde{J} \wedge \tilde{J}=\int_{\mathbb{P}\left(\nu_{0}, \ldots, \nu_{n}\right)} J \wedge J \wedge J \wedge \eta_{\mathrm{M}}
$$

using the Poincaré dual $\eta_{\mathrm{M}}=\operatorname{deg}(p) J$ of $\mathrm{M}$ in $\mathbb{P}\left(\nu_{0}, \ldots, \nu_{n}\right)$. Taking into account the correct normalisation for the Kähler form of the $\mathbb{P}\left(\nu_{0}, \ldots, \nu_{n}\right)$, namely such that $\int_{\mathbb{P}\left(\nu_{0}, \ldots, \nu_{n}\right)} J^{n}=\left(\Pi \nu_{i}\right)^{-1}$, we simply get

$$
\kappa_{0}(\tilde{J}, \tilde{J}, \tilde{J})=\frac{\operatorname{deg}(p)}{\prod_{i=0}^{n} \nu_{i}}
$$

That is, $\kappa_{0}=\{5,3,2,1\}$ for $k=\{5,6,8,10\}$.

To get the Kähler potential we use eq. (4.1) with the replacement $\left(z^{a}, \mathscr{G}_{a}\right) \rightarrow$ $\left(\omega^{a}, \mathscr{F}_{a}\right)$, or $\Pi^{\prime} \rightarrow \Pi=\left(\mathscr{F}_{1}, \mathscr{F}_{2}, \omega^{1}, \omega^{2}\right)^{\mathrm{T}}$. We find $\left(t=t_{1}+i t_{2}\right)$

$$
K_{0}=-\log \left(\frac{4}{3} \kappa t_{2}^{3}\right) \text {. }
$$

From this Kähler potential we easily derive the large radius limits of the metric $g_{i \bar{t}}^{0}=3 / 4 t_{2}^{2}$ and of the Ricci tensor $R_{t \bar{t}}^{0}=-\frac{2}{3} g_{\bar{t}}^{0}$. For the Ricci scalar one thus gets 
$R^{0}=-\frac{4}{3}$ and for the invariant Yukawa coupling $\mathscr{Y}_{0}=\frac{2}{3} \sqrt{3}$. These same constant values were found as the large complex structure limits for the $(2,1)$ moduli space of $\mathrm{M}^{\prime}$.

These infinite radius results now get modified by sigma model loops and instanton contributions, the latter being non-perturbative in the sigma model expansion parameter $1 / R^{2} \sim 1 / t, R$ being a measure for the size of the manifold. This means that the prepotential in general has the form

$$
\tilde{\mathscr{F}}=-\frac{\kappa_{0}}{6} t^{3}+\frac{1}{2} a t^{2}+b t+c+\mathrm{O}\left(\mathrm{e}^{-t}\right)
$$

leading to

$$
\Pi_{\text {pert. }}=\left(\omega^{2}\right)^{2}\left(\begin{array}{c}
-\frac{1}{2} \kappa_{0} t^{2}+a t+b \\
\frac{1}{6} \kappa_{0} t^{3}+b t+c \\
t \\
1
\end{array}\right)
$$

The polynomial part is perturbative. It is fixed by the fact that for the Yukawa couplings there is a perturbative non-renormalization theorem. Only imaginary parts of $a, b$ and $c$ affect the Kähler metric.

The instanton corrections are in general hopeless to compute directly. It is however made possible by the mirror hypothesis which implies that the two prepotentials $\mathscr{G}$ and $\mathscr{F}$ are essentially the same, but generally expressed in two different symplectic bases; i.e. $\Pi$ and $\Pi^{\prime}$ are related by a symplectic transformation and then lead to the same Kähler metric. We find an integer symplectic matrix which relates $\Pi$ and $\Pi^{\prime}$ up to a gauge transformation by relating their asymptotic limits where the limit of $\Pi^{\prime}$ is obtained from the asymptotic limit of (3.7) and that of $\Pi$ from the ansatz (5.3). We have already seen that in terms of the variable $t \propto i \log (\gamma \alpha)$ the large complex structure and large radius limits of the Kähler metrics for the moduli spaces of the $(2,1)$ and $(1,1)$ moduli agree. Fixing the asymptotic relation to $t \simeq(k / 2 \pi i) \log (\gamma \alpha)$ we find for each case an integer symplectic matrix $N$ such that $\left(1 / \omega^{2}\right) \Pi=N\left(1 / \mathscr{G}_{2}\right) \Pi^{\prime}$. This then also gives the relation between $\omega^{1}, \omega^{2}$ and $w_{0}, w_{1}, w_{2}, w_{k-1}$ and allows us to express $t$ in terms of $\alpha$. Choosing $N$ as simple as possible, we find

$$
\begin{aligned}
& N_{k=5}=\left(\begin{array}{rrrr}
-1 & 0 & 0 & 0 \\
0 & 0 & 0 & -1 \\
2 & 0 & -1 & 0 \\
0 & 1 & 0 & 0
\end{array}\right), \quad N_{k=6}=N_{k=8}=-\Sigma, \\
& N_{k=10}=\left(\begin{array}{rrrr}
-1 & 0 & 0 & 0 \\
0 & 0 & 0 & -1 \\
0 & 0 & -1 & 0 \\
0 & 1 & 0 & 0
\end{array}\right)
\end{aligned}
$$


which corresponds to the following choice of the parameters $a, b$ and $c$ in (5.3)*

$$
\{a, b, c\}= \begin{cases}\left\{-\frac{11}{2}, \frac{25}{12},-\frac{25 i}{\pi^{3}} \zeta(3)\right\} & (k=5) \\ \left\{-\frac{9}{2}, \frac{7}{4},-\frac{51 i}{2 \pi^{3}} \zeta(3)\right\} & (k=6) \\ \left\{-3, \frac{11}{6},-\frac{37 i}{\pi^{3}} \zeta(3)\right\} & (k=8) \\ \left\{-\frac{1}{2}, \frac{17}{12},-\frac{36 i}{\pi^{3}} \zeta(3)\right\} & (k=10)\end{cases}
$$

The relation between $t$ and $\alpha$ is

$$
\begin{aligned}
t=\frac{\omega^{1}}{\omega^{2}}= & -\frac{k}{2 \pi i}\{\log (\gamma \alpha) \\
& \left.+\left[\sum_{N=0}^{\infty} \frac{\underline{(k N) !}}{\prod_{i=0}^{4}\left(\nu_{i} N\right) !} \phi_{k}(N)(\gamma \alpha)^{-k N}\right] /\left[\sum_{N=0}^{\infty} \frac{(k N) !}{\prod_{i=0}^{4}\left(\nu_{i} N\right) !}(\gamma \alpha)^{-k N}\right]\right\}
\end{aligned}
$$

where the second expression is valid for $\alpha$ large. Using the known transformation $(a t)^{-1}$ on $w$ we have checked in all cases that $t(\alpha)$ transforms as $t \rightarrow t+1$ and thus $s=(a t)^{-k}: t \rightarrow t+k$ when $\alpha$ is transported around infinity. This is in accordance with the lemma in sect. 2 of ref. [14], where this fact is used to determine $t$ up to an additive constant.

Note that besides the $\mathrm{O}\left(t^{3}\right)$ term which fixes the large radius limit of the Yukawa coupling among the polynomial terms in $\tilde{\mathscr{F}}$ only the constant one contributes to the Kähler metric. It has been identified in ref. [3] with the four loop contribution calculated in ref. [36]. This term also makes its appearance in the effective low-energy string actions extracted from tree level string scattering amplitudes [37]. The exponentially small instanton corrections do affect the Yukawa

\footnotetext{
$\star$ The contribution $\alpha \zeta(3)$ arises from the terms $\alpha \phi^{\prime \prime}(0)$ in eq. (3.7).
} 
TABLE 1

Values of $n_{j}$

\begin{tabular}{|c|c|c|}
\hline & $k=5$ & $k=6$ \\
\hline$n_{0}$ & 5 & 3 \\
\hline$n_{1}$ & 2875 & 7884 \\
\hline$n_{2}$ & 609250 & 6028452 \\
\hline$n_{3}$ & 317206375 & 11900417220 \\
\hline$n_{4}$ & 242467530000 & 34600752005688 \\
\hline$n_{5}$ & 229305888887625 & 124595034333130080 \\
\hline$n_{6}$ & 248249742118022000 & 513797193321737210316 \\
\hline$n_{7}$ & 95091050570845659250 & 2326721904320912944749252 \\
\hline$n_{8}$ & 375632160937476603550000 & 11284058913384803271372834984 \\
\hline \multirow[t]{2}{*}{$n_{9}$} & 503840510416985243645106250 & 57666069759834844985369823018768 \\
\hline & $k=8$ & $k=10$ \\
\hline$n_{0}$ & 2 & \\
\hline$n_{1}$ & 29504 & 231200 \\
\hline$n_{2}$ & 128834912 & 12215785600 \\
\hline$n_{3}$ & 1423720546880 & 1700894366474400 \\
\hline$n_{4}$ & 23193056024793312 & 350154658851324656000 \\
\hline$n_{5}$ & 467876474625249316800 & 89338191421813572850115680 \\
\hline$n_{6}$ & 10807872280363954752338400 & 26107067114407746641915631734400 \\
\hline$n_{7}$ & 274144987164929172592851362112 & 8377961119575977127785199800102445600 \\
\hline$n_{8}$ & 7446718087338043414223489290659040 & 2879133858909474665080674534026982622960000 \\
\hline & 100477600893029956465355672397798401 & 29487474393188294680546419175097976102240000 \\
\hline
\end{tabular}

couplings as well as the Kähler metric. To get the Yukawa coupling we transform $\kappa_{\alpha \alpha \alpha}$ to the coordinate $t$ and find that the infinite radius value $\kappa_{0}$ gets corrected to

$$
\kappa_{t t t}=\left(\frac{\omega^{2}}{\mathscr{G}_{2}}\right)^{2} \kappa_{\alpha \alpha \alpha}\left(\frac{\mathrm{d} \alpha}{\mathrm{d} t}\right)^{3}
$$

The prefactor expresses the gauge freedom and is due to the relative factor (besides the integer symplectic matrix) between $\Pi$ and $\Pi^{\prime}$ whose components appear in the definition of the holomorphic three form which enters quadratically in $\kappa_{\alpha \alpha \alpha}$. In the gauge $\omega^{2}=1$ this becomes $\kappa_{0}+\mathrm{O}(q)$ with $q=\exp (2 \pi i t)$, where the instanton contributions come with integer coefficients. Indeed, on inverting the series (5.5) and expressing the result in the form $\kappa_{t t t}=\kappa_{0}+\sum_{j=1}^{\infty} n_{j} j^{3} q^{j} /\left(1-q^{j}\right)$ conjectured in ref. [3] and proven in ref. [38] we find the numbers $n_{j}$ which count the rational curves of degree $j$ in $\mathrm{M}$ (table 1 ) *.

* Our results agree with those of ref. [3] for the case $k=5$ and with those of ref. [14] for the cases $k=5,6,8$. Our results for $k=10$ differ by a factor of two, due to the different normalizations of the constant term. 


\section{Conclusions}

Starting from the Picard-Fuchs equations we have computed the complete expressions for the prepotential, the Kähler metric and Yukawa couplings of the heterotic string compactified on those Calabi-Yau spaces with one $(2,1)$ modulus which can be represented by one polynomial constraint in $\mathbb{P}_{4}$. We could also get the same quantities for the manifold related by mirror symmetry, where the dependence is now on the $(1,1)$ modulus and is due to instanton corrections.

One can now also study, as was done for the $k=5$ case in ref. [3], the duality symmetry of those models. One can easily generalize the procedure of ref. [3] and identify a parameter $\gamma \in \mathrm{H}^{+}$, on which the monodromy transformations on the periods are represented as a two-generator discrete subgroup of $\operatorname{SL}(2 ; \mathbb{R}) . \gamma$ is a map of the $\alpha^{k}$ plane to a pair of triangles which together constitute a fundamental region for the group of duality transformations. Since the two transformations $S$ and $t$ are of infinite order they will correspond to two parabolic elements whereas the transformation $A$ will be represented * by an elliptic element ${ }^{\star \star}$ of order $k$ [40]. Since there are only two generators these elements will not be independent. The angles of the two triangles will thus be $(0,0, \pi / k)$ corresponding to fixed points of two parabolic and one elliptic transformation. We choose these fixed points to be $i \infty, \tan (\pi \delta)$ and $i$. Here we have defined $\delta=(k-1) / 2 k$. The first two fixed points belong to two parabolic elements and the last to an elliptic element. The function $\gamma(\alpha)$ then maps the fixed points of $A, S$ and $T$ to $\gamma(0)=i$, $\gamma(\infty)=i \infty$ and $\gamma(1)=\tan (\pi \delta)$, and can be determined by standard methods [41] to be a ratio of hypergeometric functions

$$
\begin{aligned}
\gamma= & i \frac{Z_{1}-\mathrm{e}^{2 \pi i \delta} Z_{2}}{Z_{1}+\mathrm{e}^{2 \pi i \delta} Z_{2}} \\
= & \frac{i}{\pi} \tan (\pi \delta)\left\{\log \left(\alpha^{k}\right)-i \pi\right. \\
& +\left[\sum_{n=0}^{\infty} \frac{\Gamma(n+\delta) \Gamma(n+1-\delta)}{(n !)^{2} \alpha^{k n}}(2 \psi(1+n)-\psi(n+\delta)-\psi(1+n-\delta))\right] \\
& \left.\times\left[\sum_{n=0}^{\infty} \frac{\Gamma(n+\delta) \Gamma(n+1-\delta)}{(n !)^{2} \alpha^{k n}}\right]^{-1}\right\}
\end{aligned}
$$

* We use the same letters to represent the transformations on $\Pi^{\prime}$ and on $\gamma$.

** A duality group with two parabolic and one elliptic element of order 3 was observed for the one-dimensional $Z_{3}$ orbifold with a discrete Wilson line in ref. [39]. 
where the last expression is valid for $|\alpha|>1$. We have defined

$$
\begin{aligned}
& Z_{1}=\frac{\Gamma^{2}(\delta)}{\Gamma(2 \delta)} F\left(\delta, \delta, 2 \delta ; \alpha^{k}\right), \\
& Z_{2}=\frac{\Gamma^{2}(1-\delta)}{\Gamma(2(1-\delta))} \alpha F\left(1-\delta, 1-\delta, 2(1-\delta) ; \alpha^{k}\right) .
\end{aligned}
$$

Using the analytic continuation properties of the hypergeometric functions we find

$$
A=\left(\begin{array}{cc}
\cos (2 \pi \delta) & -\sin (2 \pi \delta) \\
\sin (2 \pi \delta) & \cos (2 \pi \delta)
\end{array}\right), \quad T=\left(\begin{array}{cc}
\cos (2 \pi \delta) & 2 \tan (\pi \delta) \sin ^{2}(\pi \delta) \\
-\sin (2 \pi \delta) & 1+2 \sin ^{2}(\pi \delta)
\end{array}\right)
$$

with fixed points $i$ and $\tan (\pi \delta)$, respectively and

$$
A T=\left(\begin{array}{cc}
1 & -2 \tan (\pi \delta) \\
0 & 1
\end{array}\right)
$$

with fixed point $i \infty$. The fundamental region $\Gamma$ can be chosen to be the region of the upper half-plane bounded by the two lines $|\operatorname{Re}(\gamma)|=\tan (\pi \delta)$ and two circular arcs extending from $\gamma=i$ to $\gamma= \pm \tan (\pi \delta)$ intersecting the imaginary axis at an angle of $\pi / k$ and the two boundary lines at zero angle. In fig. 1 we show on the left-hand-side these fundamental regions in the $\gamma$ plane together with its images under the operation of $A, \ldots, A^{k}$. Here and below we restrict ourselves to the cases $k=6,8,10$. For $k=5$ we refer to ref. [3].

It is possible to relate the parameters $\gamma$ and $t$ as their expansions in powers of $\alpha$ are known, and thus express the couplings on the original manifold $\mathbf{M}$ in terms of the parameter on which the duality group acts in a simple way. Using this power series expansion we can map the fundamental region from the $\gamma$ plane into the $t$ plane. The images are quadrangles whose upper corners are at $i \infty$, the lower corners corresponding to the Gepner points are at $\frac{1}{2}(-1+i \sqrt{3}), \frac{1}{2}(-1+i(1+\sqrt{2}))$ and $\frac{1}{2}(-1+i \sqrt{5+2 \sqrt{5}})$, respectively. These values follow easily from the relation between $t$ and the $w_{i}$ and their expansion for $|\alpha|<1$. The real parts of the location of the right and left corners are $-1 / 2 \pm 1 / 2$. On the right-hand-side of fig. 1 we have plotted these images of $\Gamma$ together with the images of the boundaries of the maps of $\Gamma$ in the $\gamma$ plane. For the $k=8$ and 10 case pairs of lines in the $\gamma$ plane are identified in the $t$ plane. As it is clear from the fact that these patterns do not repeat under the shift $t \rightarrow t+1$, there are points in the image of $\Gamma$ which can be identified by an $A^{m} T A^{n}$ operation in the $t$ plane. For $k=6$ and $k=10$ the images of the different fundamental regions even overlap. 

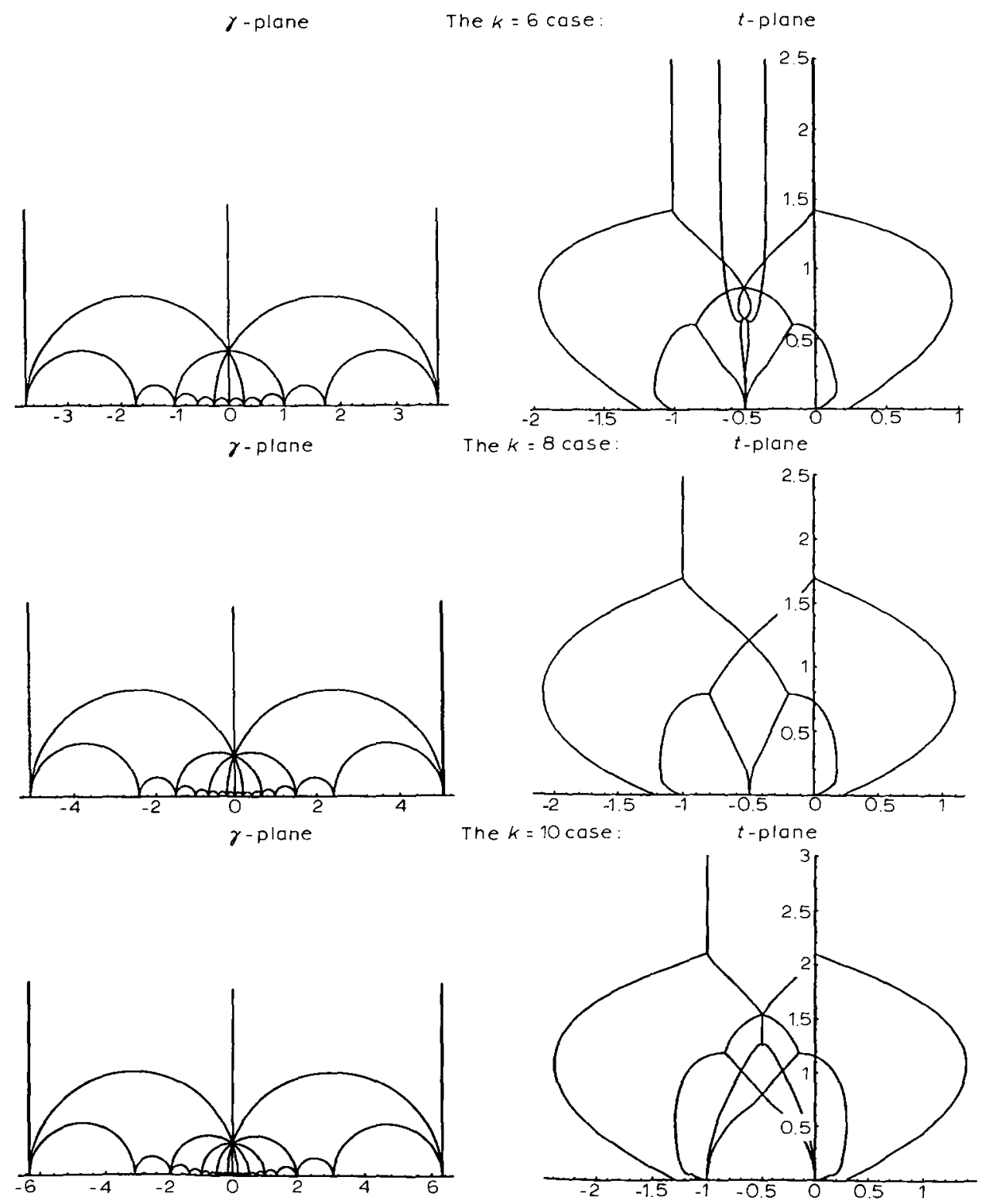

Fig. 1. Fundamental regions in the $\gamma$-planes and their images in the $t$-planes.

While it is $\gamma$ on which the duality acts simply by fractional linear transformations the $t$ parameter has a simple geometrical interpretation in that $t e=B+i J$ is the complexified Kähler form (see e.g. ref. [42]) so that the volume of the manifold scales like $\operatorname{Im}(t)^{3}=: R^{6}$. From fig. 1 it is clear that one can restrict oneself to a part of the $t$ plane which is bounded from below by a minimal length $\operatorname{Im}(t)=R^{2}$. 
The Yukawa coupling will have a simple transformation law under modular transformations. This follows from the fact that the one matter superfield which is related to the modulus $\gamma$ via world-sheet supersymmetry will transform homogeneously, and to have an invariant supergravity action the Yukawa coupling must also transform homogeneously [43]. Having computed the Yukawa couplings we thus have an explicit function of the modulus, which, when raised to the appropriate power, is also a candidate for a non-perturbative superpotential for the modulus itself. Of course, whereas for the modular group $\operatorname{SL}(2 ; \mathbb{Z})$ this function is known to be more or less unique, practically nothing is known about automorphic functions of the groups we encounter here. Work in this direction is in progress.

The models considered here represent only a very restricted class and do not lead to any even remotely realistic low energy theory. To make further progress one has to extend the analysis in several directions. One is to consider models described by higher dimensional projective varieties. There are a few examples of this kind with $h_{(2,1)}=1$, which can be studied as a first step in this direction. Another generalization is to models defined by more than one polynomial constraint. The other obvious direction to go is to consider models with more than one modulus, leading to partial differential equations for the periods. This seems to be the hardest of the possible generalizations.

Another possibility to arrive at the couplings of strings on Calabi-Yau spaces might be to follow the method of ref. [44]. In the cases we have considered here it leads to two coupled non-linear differential equations satisfied by the Kähler potential and the Kähler metric, which can be cast into the form of the SP(4) Toda equation whose solution is known.

We plan to come back to these issues in the future.

Note added: While we were in the process of writing up these results we were informed by D. Lüst and P. Candelas that A. Font has also treated the same one modulus models with similar methods [46].

We thank M.G. Schmidt for useful discussions. 


\section{Appendix A}

TABLE A.1

\begin{tabular}{|c|c|c|c|c|}
\hline & \multicolumn{4}{|c|}{$\mathrm{M}=\left\{x_{i} \in \mathbb{P}(1,1,1,1,1) \mid x_{0}^{5}+x_{1}^{5}+x_{2}^{5}+x_{3}^{5}+x_{4}^{5}=0\right\}$} \\
\hline \multicolumn{5}{|c|}{ Generators } \\
\hline 1 & & 101 & 1 & -200 \\
\hline $\mathbb{Z}_{5}$ & $(10040)$ & $49(25)$ & $5(1)$ & -88 \\
\hline $\mathbb{Z}_{5}$ & $(12340)$ & $21(21)$ & $1(1)$ & -40 \\
\hline $\mathbb{Z}_{5} \times \mathbb{Z}_{5}$ & $(10040) \times(12340)$ & $21(5)$ & $17(1)$ & -8 \\
\hline $\mathbb{Z}_{5}$ & $(12200)$ & $17(17)$ & $21(1)$ & 8 \\
\hline $\mathbb{Z}_{5} \times \mathbb{Z}_{5}$ & $(12340) \times(10220)$ & $1(1)$ & $21(1)$ & 40 \\
\hline $\mathbb{Z}_{5} \times \mathbb{Z}_{5}$ & $(10040) \times(10400)$ & $5(5)$ & $49(1)$ & 88 \\
\hline $\mathbb{Z}_{5} \times \mathbb{Z}_{5} \times \mathbb{Z}_{5}$ & $(10040) \times(10400) \times(14000)$ & $1(1)$ & $101(1)$ & 200 \\
\hline \multicolumn{5}{|c|}{$\mathrm{M}=\left\{x_{i} \in \mathbb{P}(2,1,1,1,1) \mid x_{0}^{3}+x_{1}^{6}+x_{2}^{6}+x_{3}^{6}+x_{4}^{6}=0\right\}$} \\
\hline Group & Generators & $h^{(1.2)}$ & $h^{(1,1)}$ & $x$ \\
\hline 1 & & 103 & 1 & -204 \\
\hline $\mathbb{Z}_{2}$ & $(03300)$ & $63(55)$ & $3(1)$ & -120 \\
\hline $\mathbb{Z}_{3}$ & $(02220)$ & $60(40)$ & $6(1)$ & -108 \\
\hline $\mathbb{Z}_{3}$ & $(04200)$ & $45(37)$ & $3(1)$ & -84 \\
\hline $\mathbb{Z}_{6}$ & (01005) & $43(21)$ & $7(1)$ & -72 \\
\hline $\mathbb{Z}_{2} \times \mathbb{Z}_{2}$ & $(03300) \times(03030)$ & $37(31)$ & $7(1)$ & -60 \\
\hline $\mathbb{Z}_{3}$ & $(12200)$ & $31(31)$ & $13(1)$ & -36 \\
\hline $\mathbb{Z}_{3} \times \mathbb{Z}_{3}$ & $(02220) \times(04200)$ & $30(16)$ & $12(1)$ & -36 \\
\hline $\mathbb{Z}_{6}$ & (04110) & $29(20)$ & $17(1)$ & -24 \\
\hline $\mathbb{Z}_{6} \times \mathbb{Z}_{3}$ & $(01005) \times(12200)$ & $27(7)$ & $15(1)$ & -24 \\
\hline $\mathbb{Z}_{6}$ & $(03210)$ & $23(19)$ & $11(1)$ & -24 \\
\hline $\mathbb{Z}_{6}$ & $(15320)$ & $21(17)$ & $9(1)$ & -24 \\
\hline $\mathbb{Z}_{3} \times \mathbb{Z}_{3}$ & $(02220) \times(02022)$ & $23(15)$ & $17(1)$ & -12 \\
\hline $\mathbb{Z}_{3} \times \mathbb{Z}_{3}$ & $(04020) \times(12200)$ & $19(11)$ & $13(1)$ & -12 \\
\hline $\mathbb{Z}_{2} \times \mathbb{Z}_{6}$ & $(03030) \times(21100)$ & $13(9)$ & $19(1)$ & 12 \\
\hline $\mathbb{Z}_{6} \times \mathbb{Z}_{2}$ & $(01005) \times(03300)$ & $17(11)$ & $23(1)$ & 12 \\
\hline $\mathbb{Z}_{6} \times \mathbb{Z}_{3}$ & $(03201) \times(04020)$ & $9(5)$ & $21(1)$ & 24 \\
\hline $\mathbb{Z}_{3} \times \mathbb{Z}_{6}$ & $(02022) \times(03210)$ & $11(7)$ & $23(1)$ & 24 \\
\hline $\mathbb{Z}_{6}$ & (21100) & $15(15)$ & $27(1)$ & 24 \\
\hline $\mathbb{Z}_{3} \times \mathbb{Z}_{6}$ & $(02022) \times(01005)$ & $17(8)$ & $29(1)$ & 24 \\
\hline $\mathbb{Z}_{2} \times \mathbb{Z}_{6}$ & $(03300) \times(04110)$ & $12(10)$ & $30(1)$ & 36 \\
\hline $\mathbb{Z}_{6} \times \mathbb{Z}_{6}$ & $(01005) \times(03210)$ & $13(3)$ & $31(1)$ & 36 \\
\hline $\mathbb{Z}_{3} \times \mathbb{Z}_{3} \times \mathbb{Z}_{3}$ & $(02220) \times(02022) \times(02202)$ & $7(7)$ & $37(1)$ & 60 \\
\hline $\mathbb{Z}_{3} \times \mathbb{Z}_{6}$ & $(02220) \times(01005)$ & $7(7)$ & $43(1)$ & 72 \\
\hline $\mathbb{Z}_{6} \times \mathbb{Z}_{6}$ & $(04110) \times(04101)$ & $3(3)$ & $45(1)$ & 84 \\
\hline $\mathbb{Z}_{6} \times \mathbb{Z}_{6}$ & $(01005) \times(01050)$ & $6(4)$ & $60(1)$ & 108 \\
\hline $\mathbb{Z}_{3} \times \mathbb{Z}_{3} \times \mathbb{Z}_{6}$ & $(02220) \times(02022) \times(01005)$ & $3(3)$ & $63(1)$ & 120 \\
\hline $\mathbb{Z}_{3} \times \mathbb{Z}_{6} \times \mathbb{Z}_{6}$ & $(02220) \times(01005) \times(01050)$ & $1(1)$ & $103(1)$ & 204 \\
\hline
\end{tabular}


TABI.E A.1 (continued)

\begin{tabular}{|c|c|c|c|c|}
\hline \multirow[b]{2}{*}{ Order } & \multicolumn{3}{|c|}{$\mathrm{M}=\left\{x_{i} \in \mathbb{P}(4,1,1,1,1) \mid x_{0}^{2}+x_{1}^{8}+x_{2}^{8}+x_{3}^{8}+x_{4}^{8}=0\right\}$} & \multirow[b]{2}{*}{$x$} \\
\hline & Generators & $h^{(1,2)}$ & $h^{(1,2)}$ & \\
\hline 1 & & 149 & 1 & -296 \\
\hline $\mathbb{Z}_{8}$ & $(01511)$ & $106(85)$ & $2(1)$ & -208 \\
\hline $\mathbb{Z}_{2}$ & $(04400)$ & $83(77)$ & $3(1)$ & -160 \\
\hline $\mathbb{Z}_{2} \times \mathbb{Z}_{8}$ & $(04400) \times(01511)$ & $69(49)$ & $5(1)$ & -128 \\
\hline $\mathbb{Z}_{4}$ & $(04220)$ & $49(39)$ & $9(1)$ & -80 \\
\hline $\mathbb{Z}_{2} \times \mathbb{Z}_{2}$ & $(04400) \times(04040)$ & $47(41)$ & $7(1)$ & -80 \\
\hline $\mathbb{Z}_{8}$ & $(07531)$ & $43(39)$ & $3(1)$ & -80 \\
\hline $\mathbb{Z}_{8}$ & $(01007)$ & $43(21)$ & $11(1)$ & -64 \\
\hline $\mathbb{Z}_{4} \times \mathbb{Z}_{8}$ & $(04220) \times(01511)$ & $41(25)$ & $9(1)$ & -64 \\
\hline $\mathbb{Z}_{8}$ & $(03311)$ & $35(35)$ & $19(1)$ & -32 \\
\hline $\mathbb{Z}_{8} \times \mathbb{Z}_{8}$ & $(01007) \times(01511)$ & $33(13)$ & $17(1)$ & -32 \\
\hline $\mathbb{Z}_{4} \times \mathbb{Z}_{8}$ & $(04022) \times(03311)$ & $29(23)$ & $21(1)$ & -16 \\
\hline $\mathbb{Z}_{4} \times \mathbb{Z}_{2}$ & $(04220) \times(04400)$ & $27(21)$ & $19(1)$ & -16 \\
\hline $\mathbb{Z}_{\mathrm{B}}$ & $(05210)$ & $25(19)$ & $17(1)$ & -16 \\
\hline $\mathbb{Z}_{8}$ & $(04310)$ & $23(19)$ & $15(1)$ & -16 \\
\hline $\mathbb{Z}_{8} \times \mathbb{Z}_{8}$ & $(02330) \times(01511)$ & $17(11)$ & $25(1)$ & 16 \\
\hline $\mathbb{Z}_{8} \times \mathbb{Z}_{8}$ & $(04301) \times(05210)$ & $15(11)$ & $23(1)$ & 16 \\
\hline $\mathbb{Z}_{4} \times \mathbb{Z}_{2} \times \mathbb{Z}_{8}$ & $(04022) \times(04040) \times(03311)$ & $19(15)$ & $27(1)$ & 16 \\
\hline $\mathbb{Z}_{4} \times \mathbb{Z}_{8}$ & $(04220) \times(04310)$ & $21(11)$ & $29(1)$ & 16 \\
\hline $\mathbb{Z}_{8}$ & $(02330)$ & $17(17)$ & $33(1)$ & 32 \\
\hline $\mathbb{Z}_{8} \times \mathbb{Z}_{8}$ & $(01007) \times(04310)$ & $19(5)$ & $35(1)$ & 32 \\
\hline $\mathbb{Z}_{B} \times \mathbb{Z}_{2}$ & $(02330) \times(04400)$ & $9(9)$ & $41(1)$ & 64 \\
\hline $\mathbb{Z}_{8} \times \mathbb{Z}_{2}$ & $(01007) \times(02330)$ & $11(11)$ & $43(1)$ & 64 \\
\hline $\mathbb{Z}_{8} \times \mathbb{Z}_{8}^{2}$ & $(02303) \times(05210)$ & $3(3)$ & $43(1)$ & 80 \\
\hline $\mathbb{Z}_{4} \times \mathbb{Z}_{4} \times \mathbb{Z}_{8}$ & $(04220) \times(04022) \times(03311)$ & $7(7)$ & $47(1)$ & 80 \\
\hline $\mathbb{Z}_{x} \times \mathbb{Z}_{x} \times \mathbb{Z}_{2}$ & $(02303) \times(01070) \times(04400)$ & $9(7)$ & $49(1)$ & 80 \\
\hline $\mathbb{Z}_{x} \times \mathbb{Z}_{4}$ & $(01070) \times(04220)$ & $5(5)$ & $69(1)$ & 128 \\
\hline $\mathbb{Z}_{8} \times \mathbb{Z}_{8} \times \mathbb{Z}_{4}$ & $(01070) \times(02303) \times(04220)$ & $3(3)$ & $83(1)$ & 160 \\
\hline $\mathbb{Z}_{8} \times \mathbb{Z}_{8}$ & $(01007) \times(01070)$ & $2(2)$ & $106(1)$ & 208 \\
\hline $\mathbb{Z}_{8} \times \mathbb{Z}_{8} \times \mathbb{Z}_{8}$ & $(01007) \times(01070) \times(01700)$ & $1(1)$ & $149(1)$ & 296 \\
\hline
\end{tabular}

TABle A.1 (continued)

\begin{tabular}{|c|c|c|c|c|}
\hline Order & $\begin{array}{c}\mathrm{M}=\left\{x_{i} \in \mathbb{P}(5,2,1,\right. \\
\text { Generators }\end{array}$ & $\begin{array}{c}x_{1}^{5}+x_{2}^{10} \\
h^{(1,2)}\end{array}$ & $\begin{array}{l}\frac{10)}{\left.4^{4}=0\right\}} \\
h^{(1, \overline{1})}\end{array}$ & - \\
\hline 1 & & 145 & 1 & -288 \\
\hline $\mathbb{Z}_{2}$ & $(00550)$ & $99(81)$ & $3(1)$ & -192 \\
\hline $\mathbb{Z}_{2} \times \mathbb{Z}_{2}$ & $(00550) \times(00055)$ & $67(49)$ & $7(1)$ & -120 \\
\hline $\mathbb{Z}_{5}$ & $(01440)$ & $47(31)$ & $11(1)$ & -72 \\
\hline $\mathbb{Z}_{5}$ & $(00280)$ & $37(29)$ & $13(1)$ & -48 \\
\hline $\mathbb{Z}_{10}$ & $(00109)$ & $39(17)$ & $15(1)$ & -48 \\
\hline $\mathbb{Z}_{10}$ & $(01350)$ & $29(17)$ & $17(1)$ & -24 \\
\hline $\mathbb{Z}_{10}$ & $(01170)$ & $17(15)$ & $29(1)$ & 24 \\
\hline $\mathbb{Z}_{10}$ & $(02330)$ & $15(15)$ & $39(1)$ & 48 \\
\hline $\mathbb{Z}_{2} \times \mathbb{Z}_{10}$ & $(00055) \times(01350)$ & $13(9)$ & $37(1)$ & 48 \\
\hline $\mathbb{Z}_{10} \times \mathbb{Z}_{2}$ & $(00109) \times(00550)$ & $11(9)$ & $47(1)$ & 72 \\
\hline $\mathbb{Z}_{5} \times \mathbb{Z}_{5}^{2}$ & $(00280) \times(00208)$ & $7(7)$ & $67(1)$ & 120 \\
\hline $\mathbb{Z}_{10} \times \mathbb{Z}_{5}$ & $(00109) \times(00280)$ & $3(3)$ & $99(1)$ & 192 \\
\hline $\mathbb{Z}_{10} \times \mathbb{Z}_{10}$ & $(00109) \times(00190)$ & $1(1)$ & $145(1)$ & 288 \\
\hline
\end{tabular}




\section{Appendix B}

We follow ref. [3] and compute two of the periods explicitly in a symplectic basis thus identifying with two particular solutions of the period equations. The remaining two are then determined by requiring that the monodromy transformations are represented as $\mathrm{Sp}(4 ; \mathbb{Z})$. To do this we represent the periods as integrals of the holomorphic three form over the cycles of the Calabi-Yau manifold. We take $\Omega$ in the gauge *

$$
\Omega(\alpha)=k \alpha \frac{x_{4} \mathrm{~d} x_{0} \wedge \mathrm{d} x_{1} \wedge \mathrm{d} x_{2}}{\partial W / \partial x_{3}}
$$

$\Omega$ can be expanded in a cohomology basis as $\Omega=z^{a} \alpha_{a}-\mathscr{G}_{a} \beta^{a}$ where the forms $\alpha_{a}$ and $\beta^{a}$ are dual to the integer symplectic homology basis $A^{a}$ and $B_{a}$ which satisfies $A^{a} \cap B_{b}=\delta_{b}^{a}, A^{a} \cap A^{b}=B_{a} \cap B_{b}=0$ and $\int_{A^{a}} \alpha_{b}=\int_{B_{a}} \beta^{b}=\delta_{a}^{b}$ with the other integrals vanishing. With this the periods are $z^{a}=\int_{A^{a}} \Omega, \mathscr{G}_{a}=\int_{B_{a}} \Omega$. We now integrate the holomorphic three-form $\Omega(\alpha)$ over the two cycles $A^{2}$ and $B_{2}$ which are close to the node and which have intersection number one [3]. They are defined as

$$
\begin{aligned}
& A^{2}=\left\{x_{k} \mid x_{4}=1, x_{i} \text { real, } x_{3} \text { s.t. } W(x)=0 \cong S^{3} \text { as } \alpha \rightarrow 1\right\}, \\
& B_{2}=\left\{x_{k}\left|x_{4}=1,\right| x_{0}|=| x_{1}|=| x_{2} \mid=\delta, x_{3} \rightarrow 0 \text { as } \alpha \rightarrow \infty\right\} .
\end{aligned}
$$

To get the correct normalization for the periods we work in a coordinate patch where one of the coordinates with weight one is set to one. This completely fixes the equivalences in $\mathbb{P}_{4}$.

$z^{2}(\alpha)$ : We will only need $z^{2}$ to lowest order in $\epsilon^{2}:=(\alpha-1)$. For points close to the node we set $x_{i}=1+y_{i}(i=0,1,2,3)$ with $y_{i}=O(\epsilon)$ and define $\partial W / \partial x_{3}=k(k$ $-1) w_{3}+\mathrm{O}\left(\epsilon^{2}\right)$ where $w_{4}$ is linear in the $y_{i}$. One then finds that $W=0$ corresponds, upon dropping terms $\mathrm{O}\left(\epsilon^{3}\right)$, to $\left(w_{3}^{2}+B_{i j} y_{i} y_{j}\right)=(2 k /(k-1)) \epsilon^{2}$, where $B_{i j}$ is a real symmetric matrix with positive eigenvalues. Converting to spherical coordinates one then finds that

$$
\begin{aligned}
z^{2}(\alpha) & =\int_{A^{2}} \Omega=\frac{4 k \pi^{2}}{(k-1)^{2}}(\operatorname{det} B)(\alpha-1)+\ldots \\
& =\frac{4 \pi^{3}}{k^{3 / 2}}\left(\prod_{i=0}^{4} \nu_{i}\right)^{1 / 2}(\alpha-1)+\mathrm{O}\left((\alpha-1)^{2}\right) .
\end{aligned}
$$

\footnotetext{
* For the relation of the above form of the holomorphic three form with the one implicit in our discussion at the beginning of sect. 3 , we refer to ref. [45].
} 
This shows that $z^{2}$ is the solution of the period equation with index one at $\alpha=1$ which is free from logarithms.

$G_{2}(\alpha)$ : We define $\xi$ via $x_{3}=\left(\alpha x_{0} x_{1} x_{2}\right)^{1 /(k-1)} \xi$ in terms of which $W=0$ reads

$$
\xi=\epsilon+\xi^{k} / k \text { with } \epsilon=\frac{1+W_{0}-x_{3}^{k}-x_{4}^{k}}{k\left(\alpha x_{0} x_{1} x_{2}\right)^{k /(k-1)}} .
$$

The holomorphic three-form then becomes

$$
\Omega=\frac{\mathrm{d} x_{0} \wedge \mathrm{d} x_{1} \wedge \mathrm{d} x_{2}}{x_{0} x_{1} x_{2}\left(\xi^{k-1}-1\right)} .
$$

Expanding $\left(1-\xi^{k-1}\right)^{-1}$ in powers of $\epsilon$ and performing the integrals over $x_{i}$ with $\left|x_{i}\right|=\delta(i=0,1,2)$ one finds *

$$
\mathscr{G}_{2}=\frac{(2 \pi i)^{3}}{\operatorname{ord} G} \sum_{m=0}^{\infty} \frac{(k m) !}{\prod_{i=0}^{4}\left(m \nu_{i}\right) !}(\gamma \alpha)^{-k m} .
$$

Candelas et al. [3] argue that under transport about $\alpha=1$ only the cycle $B_{2}$ transforms non-trivially in that it picks up a multiple of $\mathrm{A}^{2}$. This means that the period $\mathscr{G}_{2}$ will pick a multiple of the period $z^{2}$. This leads to the identification of $\tilde{w}(\alpha)$ in eq. (3.8) and the assertion that the other periods are free from logarithms.

\section{References}

[1] K. Kikkawa and M. Yamasaki, Phys. Lett. B149 (1984) 357;

N. Sakai and I. Senda, Progr. Theor. Phys. 75 (1984) 692;

V.P. Nair, A. Shapere, A. Strominger and F. Wilczek, Nucl. Phys. B287 (1987) 402;

A. Giveon, E. Rabinovici and G. Veneziano, Nucl. Phys. B322 (1989) 167

[2] M. Dine, P. Huet and N. Seiberg, Nucl. Phys. B322 (1989) 301;

J. Lauer, J. Mas and H.P. Nilles, Phys. Lett. B226 (1989) 251;

M. Spaliński, Phys. Lett. B275 (1992) 47

[3] P. Candelas, X. de la Ossa, P. Green and L. Parkes, Nucl. Phys. B359 (1991) 21

* Here we use that given $\xi=\epsilon+\xi^{k} / k$,

$$
\frac{1}{1-\xi^{k-1}}=\sum_{m=0}^{\infty}\left(\begin{array}{c}
m k \\
m
\end{array}\right) \frac{1}{k^{m}} \epsilon^{m(k-1)}
$$

This is easily shown as follows: one makes the ansatz $\left(1-\xi^{k-1}\right)^{-1}=\sum_{\nu=0}^{\infty} a_{\nu} \epsilon^{\nu}$. Noting that

$$
1-\xi^{k-1}=\partial \epsilon(\xi) / \partial \xi \text { and } a_{\nu}=\oint \frac{\mathrm{d} \epsilon}{1-\xi^{\nu+1}} \frac{1}{\epsilon^{\nu+1}}=\oint \frac{\mathrm{d} \xi}{\epsilon^{\nu+1}}
$$

we get the result upon expanding $\left(\epsilon^{\nu+1}\right)^{-1}$ in powers of $\xi$ and extracting the residue. 
[4] S. Ferrara, D. Lüst, A. Shapere and S. Theisen, Phys. Lett. B225 (1989) 363

[5] L. Dixon, D. Friedan, E. Martinec and S. Shenker, Nucl. Phys. B282 (1987) 13;

S. Hamidi and C. Vafa, Nucl. Phys. B279 (1987) 465;

J. Lauer, J. Mas and H.P. Nilles, Nucl. Phys. B351 (1991) 362

[6] D. Gepner, Nucl. Phys. B296 (1988) 757; $N=2$ String Theory; Lectures at the Trieste spring school on superstrings (1989), PUPT-1121

[7] L. Ibanez and D. Lüst, Duality anomaly cancellation, minimal string unification and the effective low-energy lagrangian of 4D strings, preprint CERN-TH-6380-92

[8] N. Seiberg, Nucl. Phys. B303 (1988) 286;

S. Cecotti, S. Ferrara and L. Girardello, Int. J. Mod. Phys. A4 (1989) 2475;

L. Dixon, V. Kaplunovsky and J. Louis, Nucl. Phys. B329 (1990) 27

[9] For reviews see S. Ferrara, Mod. Phys. Lett. A6 (1991) 2175;

S. Ferrara and S. Theisen, Proc. Hellenic Summer School 1989 (World Scientific Singapore);

A. Strominger, Commun. Math. Phys. 133 (1990) 163;

P. Candelas and X. de la Ossa, Nucl. Phys. B355 (1991) 455

[10] J. Distler and B. Greene, Nucl. Phys. B309 (1988) 295

[11] B. Greene and M. Plesser, Nucl. Phys. B338 (1990) 15

[12] P. Candelas, M. Lynker and R. Schimmrigk, Nucl. Phys. B341 (1990) 383

[13] J. Fuchs, A. Klemm, Ch. Scheich and M.G. Schmidt, Phys. Lett. B232 (1989) 317, Ann. Phys. 204 (N.Y.) (1990) 317

[14] D.R. Morrison, Picard-Fuchs equations and mirror maps for hypersurfaces, preprint DUK-M-91-14

[15] A. Strominger and E. Witten, Commun. Math. Phys. 101 (1985) 341

[16] D. Gepner, Nucl. Phys. B296 (1988) 757; N=2 string theory; Lectures at the Trieste spring school on superstrings (1989) PUPT-1121

[17] A. Klemm and R. Schimmrigk, Landau-Ginzburg string vacua, preprint CERN-TH-6459/92, TUM-TP-142/92

[18] A. Lütken and G. Ross, Phys. Lett. B213 (1998) 152

[19] L. Dixon, J.A. Harvey, C. Vafa and E. Witten, Nucl. Phys. B261 (1985) 678

[20] P.S. Aspinwall, A. Lütken and G. Ross, Phys. Lett. B241 (1990) 373

[21] A. Klemm and M.G. Schmidt, Phys. Lett. B245 (1990) 53;

J. Fuchs, A. Klemm and M.G. Schmidt, Ann. Phys. (N.Y.) 214 (1992) 221

[22] A. Fuijki, Publ. RIMS, Kyoto Univ. 10 (1974) 293

[23] S. Roan and S.T. Yau, Acta Math. Sinica 3 (1987) 256

[24] A. Klemm, Stringkompaktifizieuring durch Tensorprodukte minimaler $n=2$ superkonformer Feldtheorein und ihre geometrische Interpretation, Ph.D. Dissertation, Heidelberg University (1990)

[25] C. Vafa, Mod. Phys. Lett. A12 (1989) 1169, Mod. Phys. Lett. A17 (1989) 1615

[26] P. Zoglin, Phys. Lett. B218 (1989) 444

[27] S.S. Roan, Int. J. Math. 1 (1990) 211; see also Topological couplings of Calabi-Yau Orbifolds, Preprint MPI/92/22

[28] P. Berglund, B.R. Greene and T. Hübsch, Classical vs. Landau-Ginzburg geometry of compactifications., preprint CERN-TH-3681-92

[29] W. Lerche, D.-J. Smit and N. Warner, Nucl. Phys. B372 (1992) 87

[30] A. Cadavid and S. Ferrar, Phys. Lett. B267 (1991) 193;

S. Ferrara and J. Louis, Phys. Lett. B278 (1992) 240;

A. Ceresole, R. D'Auria, S. Ferrara, W. Lerche and J. Louis, Picard-Fuchs equations and special geometry; preprint CERN-TH.6441/92

[31] A. Klemm, M.G. Schmidt and S. Theisen, Int. J. Mod. Phys. A7 (1992) 6215

[32] A. Klemm and S. Theisen; unpublished

[33] E.L. Ince, Ordinary differential equations (Dover, 1956)

[34] J. Distler and B. Greene, Nucl. Phys. B309 (1988) 295

[35] S.-T. Yau, Compact three dimensional Kähler manifolds with zero Ricci curvature in Geometry, Anomalies, Topology, Ed. W. Bardeen and A. White (World Scientific, Singapore, 1985) 
[36] M. Grisaru, A. van de Ven and D. Zanon, Phys. Lett. B173 (1986) 423, Nucl. Phys. B277 (1986) 388,409

[37] D. Gross and J. Sloan, Nucl. Phys. B291 (1987) 41;

N. Cai and C. Nunez, Nucl. Phys. B287 (1987) 279

[38] P.S. Aspinwall and D.R. Morrison, Topological field theory and rational curves, preprint OUTP91-32P

[39] J. Erler, Untersuchungen von Moduliräumen in Stringtheorien, Ph.D. Thesis TU-München, preprint MPI-Ph/92-21

[40] A good reference on this subject is e.g. J. Lehner, A short course in automorphic functions (Holt, Rinehart and Winston, 1966)

[41] See, e.g. F. Klein und R. Fricke, Vorlesungen über die Theorie der elliptischen Modulfunktionen, Vol. I (Teubner, 1890)

[42] P. Candelas and X. de la Ossa, Nucl. Phys. B355 (1991) 455

[43] S. Ferrara, D. Lüst and S. Theisen, Phys. Lett. B233 (1989) 147

[44] S. Cecotti and C. Vafa, Nucl. Phys. B367 (1991) 359

[45] P. Candelas, Nucl. Phys. B298 (1988) 458

[46] A. Font, Periods and duality symmetries in Calabi-Yau compactifications, preprint UCVFC-1-92 